Background: Procedures applied by Polish military surgical teams during foreign missions conducted from 2008 to 2013 (total number of trauma patients $-1,327$ ), specifically in terms of treating locals, and the operations of the Urban SAR Groups of the Polish State Fire Service during earthquake-related rescue missions from 1999 to 2014, were assessed.

Methods: Medical procedures applied by the Polish military surgical teams and the Urban SAR Groups were analyzed, specifically in terms of using their experience to improve the efficiency of medical treatment of disaster victims.

Results: The operations of the level-2 surgical teams in Afghanistan have greatly advanced knowledge of dealing with trauma victims with limited personnel and restricted transport resources. The challenges involved in treating local patients always include limited options of long-term observation, and treatment which necessitates modification of treatment methods. Based on the experience of the Urban SAR groups acquired during post-earthquake rescue efforts, there is significant need for more extensive medical aid, specifically in cases of dealing with damage to the extremities, wound treatment and the "crash syndrome". Experience of and procedures followed by the level-2 surgical teams in the course of damage control surgery and damage control orthopaedics, may be directly applicable to treating disaster victims, and also if there is no continued observation of victims.

Conclusion: Damage control surgery procedures may be applied to treatment of disaster victims. However, methods and standards of treatment must be carefully tailored to the inability to provide long-term care and patients relying on local healthcare services for continued treatment. That is specifically important in case of orthopedic trauma treatment procedures.

Prehosp Disaster Med 2017;32(Suppl. 1):s123-s124

doi:10.1017/S1049023X17003508

\section{Emergency Teams in Cascading Disasters}

Patrizia I. Duda ${ }^{1}$, Eran Oren ${ }^{2}$

1. Institute For Risk and Disaster Reduction, University College London (UCL), London/United Kingdom

2. Weizmann Institute of Science, Rehovot/Israel

Study/Objective: To analyze the role of non-medical disaster response teams in cascading disasters, and their fit within wider Disaster Risk Reduction and Response (DRR\&R) efforts.

Background: The field of disaster studies has recently seen a focus on so-called "cascading disasters." What is meant is disasters with cascading effects across functional and national boundaries, leading to secondary disasters of a similar or larger magnitude than the initial event (Pescaroli and Alexander, 2015). The notion of cascades points our focus to an important question within current disaster response: Are we sending the right people? Put differently, is the composition of our disaster response teams fit for DRR\&R in cascading disasters? In this regard, the role and potential of nonmedical personnel to prevent, stop, and respond to cascading disasters has received little attention. Yet, considering how cascading disasters spread across critical infrastructures, such as electrical, transportation, or sewerage systems, clearly the focus on sending predominantly medical teams to disaster zones is insufficient.
Methods: This study is conducted in in three steps: 1 . An extensive literature review. 2. 20 in-depth, semi-structured interviews with: a) non-medical key personnel in areas such as construction, municipal planning, and the electrical grid to understand their perceptions of their role and abilities within DRR\&R in cascading disasters. and b) key personnel from international DRR\&R teams to understand their perceptions of the role of non-medical personal in cascading disasters.

Results: The insights of the literature review and interviews will be analyzed and consolidated into meaningful conclusions and actionable recommendations.

Conclusion: The research aims to suggest improved compositions of response teams that may prevent deterioration in disasters scenarios rather than focusing on the initial disaster situation alone. Final conclusions will be presented at the 2017 WADEM conference in Toronto.

Prehosp Disaster Med 2017;32(Suppl. 1):s124

doi:10.1017/S1049023X1700351X

\section{Medical Response to the 2016 Fort McMurray Wildfires - Descriptive Epidemiology of Patients Presenting to a Field Hospital \\ Dirk Chisholm, Joshua Bezanson, Kevin Hanrahan, Gwynn Curran-Sills \\ Medical, Canada Task Force 2, Calgary/AB/Canada}

Study/Objective: To describe the epidemiology of patient presentation to a physician, nurse, and paramedic staffed field hospital during the 2016 Fort McMurray Wildfires (FMMW). Background: The FMMW was the most economically devastating natural disaster in Canadian history, resulting in the evacuation of over 80,000 citizens, burning of over 1,600 structures, with a cost of over $\$ 9$ billion CDN. Canada Task Force 2 (CAN-TF2) is Alberta's all-hazards disaster response team, which includes Heavy Urban Search and Rescue (HUSAR) and Disaster Medical Assistance Team (DMAT) capabilities. As part of CAN-TF2's deployment, a field hospital was established to support the incident as a result of the evacuation of local healthcare facilities.

Methods: A retrospective chart review was conducted of all Patient Care Reports from the field hospital to determine chief complaint, organized by Canadian Emergency Department Information System (EDIS) Presenting Complaint List. Disposition and patient demographics were also recorded.

Results: A total of 162 patients were seen over a 14-day period. Medical force protection accounted for 32/162 (20\%) of patient presentations, with the remainder being patients external to CANTF. Evacuation to higher levels of care was required for 23/162 (14\%) patients. Table 1 describes presenting complaints. The leading presenting complaint was prescription / medication request $(n=47)$, followed by foreign body eye injury $(n=14)$, GI complaints $(n=11$ and $n=9)$, and foot care $(n=9)$.

Conclusion: The majority of patients presented with primary care complaints. While CAN-TF2's primary mission was to provide medical force protection, most of the patients treated were external to the agency. Of the incident responders who 
presented for care, the majority were able to return to work. A major medical challenge encountered was responding to a serious GI illness outbreak. Future medical planning will focus on provision of pharmacy services and promoting the use of eye personal protective equipment in wildfire hazard zones.

Prehosp Disaster Med 2017;32(Suppl. 1):s124-s125

doi:10.1017/S1049023X17003521

Discovering Best Practice for the Implementation of Evacuation Centers for Vulnerable Populations: Findings from a Japanese Pilot Study Mayumi Kako ${ }^{1}$, Yosuke Takada ${ }^{2}$, Paul Arbon ${ }^{3}$, Malinda Steenkamp ${ }^{4}$, Benjamin J. Ryan ${ }^{5}$

1. School Of Nursing and Midwifery, Flinders University, Torrens Resilience Institute, Adelaide/SA/Australia

2. Disaster Reduction and Human Renovation Institution, Kobe/ Japan

3. Torrens Resilience Institution, Adelaide/SA/Australia

4. Torrens Resilience Institute, Adelaide/ACT/Australia

5. College Of Public Health, Medical and Veterinary Sciences, James Cook University, Townsville/QLD/Australia

Study/Objective: This paper will report the preliminary findings of a pilot study, undertaken with local government officials in Japan, concerning their involvement in planning for, setting up, and managing evacuation centers for vulnerable populations in Japan during the Great East Japan Earthquake in 2011. The objective is to illuminate the challenges that officials faced, and the resolutions and lessons learned in the preparation of evacuation centers through this event.

Background: Potentially vulnerable population groups in disasters include the elderly and frail, people who are isolated, and those with chronic diseases including mental health conditions or mobility issues. The 2011 Great East Japan Earthquake disaster affected regions of Japan where the proportion of older population is relatively higher than other parts of the country. In 2008, the Japanese Government Cabinet Office implemented guidelines for the preparation and establishment of evacuation centers for vulnerable populations. However, the 2011 disaster highlighted issues regarding the role and responsibility across governments relating to planning, setup, and management of evacuation centers.

Methods: The study was comprised of two phases. The first involved interviews with local government and relevant agencies' officials who have been involved in establishing evacuation centers for vulnerable populations in Japan. Five officials were recruited from the local government area affected by the disaster in Japan. Face-to-face, semi-structured interviews were audio-recorded and thematic analysis was conducted using NVivo software.

Results: Four themes emerged. They were: (1) reflecting on role and responsibility for community, (2) awareness of the need for preparedness, (3) factors causing organizations to be underprepared, and (4) the need for greater community resilience. Conclusion: This pilot study demonstrated that the establishment of clear role descriptions and responsibilities are key for local governments to prepare for the establishment of disaster evacuation centers, particularly for vulnerable populations.

Prehosp Disaster Med 2017;32(Suppl. 1):s125

doi:10.1017/S1049023X17003533
Hospital Surge Capacity in the 2011 Great East Japan

Earthquake and Tsunami

Kazuma Morino

Emergency, Yamagata Prefectural Medical Center for Emergency, Yamagata/Japan

Study/Objective: Until now, there is no experience or evidence about hospital surge capacity in Tsunami disasters in Japan. In the meantime, we had experienced the 2011 Great East Earthquake and Tsunami. So, we will investigate how we make hospital surge capacity in Tsunami disasters.

Background: Surge capacity is a functional expansion capability for catastrophic situations within the organization to deal with a disaster. For hospitals, it can be said that it is the ability of the health care system to accept a large number of patients that occur in a sudden disaster. Not just one of the hospitals, the hospital group, the first-aid station in the area, and more must be considered, as well as the ability of the health care system in the affected prefecture, neighboring prefectures, and nationwide.

Methods: We have investigated five hospitals in Miyagi Prefecture. All hospitals are disaster-based hospitals that were prepared for natural disasters and designated by the local government. We compared bed capacity of these hospitals at peacetime and at the time of disaster; how they effected their surge capacity, and the regional bed capacity. We studied bed capacity in Yamagata Prefecture and places next to Miyagi Prefecture at that time.

Results: Two of the five hospitals that were near the pacific coast should install additional (extra) beds. The number of beds were about two or three times short of daily new admissions. Another two of five that were placed at inland hospitals had no need for additional beds. All hospitals stopped ordinary work to make or expand their capacity of beds and medical staff. Yamagata Prefecture could make slightly more bed capacity.

Conclusion: Except big hospitals in the affected area by Tsunami, hospitals were required to expand their additional (extra) beds for two or three times the daily new admissions, medical staff, and equipment suitable for disaster situations.

Prehosp Disaster Med 2017;32(Suppl. 1):s125

doi:10.1017/S1049023X17003545

Evacuation Burden of a Safety-Net Academic Medical Center during Hurricane Sandy: Implications for

Reverse Triage

Christopher P. Wang ${ }^{1}$, Rushabh Shab ${ }^{2}$, Sidrah Malik', Ian Portelli ${ }^{1}$, Lewis R. Goldfrank ${ }^{1}$, Silas W. Smith ${ }^{1}$

1. Ronald O. Perelman Department Of Emergency Medicine, New York University School of Medicine, New York/NY/United States of America

2. New York University School of Medicine and Stern School of Business, New York/NY/United States of America

Study/Objective: We describe evacuation burdens of a municipal, safety-net academic medical center, following the largest Atlantic hurricane in United States history.

Background: Typically applied to hospitals receiving surge capacity, reverse triage models have suggested that up to 